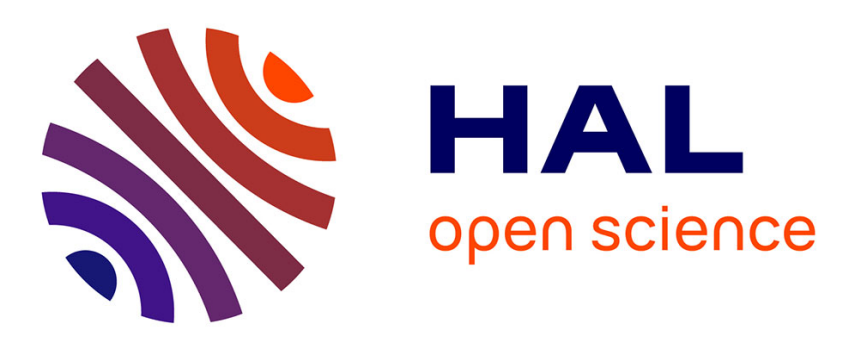

\title{
Market Efficiencies and Market Risks
}

Pierre-André Maugis

\section{To cite this version:}

Pierre-André Maugis. Market Efficiencies and Market Risks. 2010. halshs-00544324

\section{HAL Id: halshs-00544324 \\ https://shs.hal.science/halshs-00544324}

Submitted on 7 Dec 2010

HAL is a multi-disciplinary open access archive for the deposit and dissemination of scientific research documents, whether they are published or not. The documents may come from teaching and research institutions in France or abroad, or from public or private research centers.
L'archive ouverte pluridisciplinaire HAL, est destinée au dépôt et à la diffusion de documents scientifiques de niveau recherche, publiés ou non, émanant des établissements d'enseignement et de recherche français ou étrangers, des laboratoires publics ou privés. 


\section{Documents de Travail du Centre d'Economie de la Sorbonne}

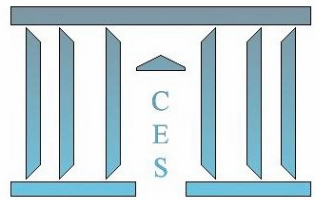

Market Efficiencies and Market Risks

Pierre-André MAUGIS

2010.94

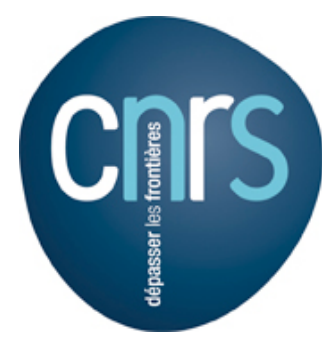




\title{
Market Efficiencies and Market Risks
}

\author{
P.A. Maugis - Paris School of Economics
}

November 22, 2010

\begin{abstract}
In recent years numerous papers constructed or simulated financial markets at an agent level, aiming to explain the non-stationarity of price processes. All such papers agree that the heterogeneity of agents and of pricing models creates a dynamics in terms of pricing models used that explains not only the non-stationarity of price processes, but also stylised facts such as bubbles and fat tails. However, all these results issue from very specific parametric set-ups, and even if multiple approaches confirm it, there is no proof of the aforementioned results outside of such specifications. By modeling agents as black boxes that receive information that they transform into an output information, information according to which they then act upon the financial market, we show that the diversity of agents is directly associated to the resulting quality of the information efficiency of the market: homogenous agents lead to good information propagation but poor information aggregation by the price, while heterogenous agents lead to good information aggregation but poor information propagation. This difference in quality of efficiency explains, outside of any parametric model, the dynamics of the number of different pricing models used within artificial stock markets.
\end{abstract}

Keywords: Market Efficiency, Group Learning and Evolutionary Games. JEL: JELG14, JELG10, JELG01 and JELC73.

\section{Introduction}

Many approaches have been considered to explain the randomness and unpredictability of prices in financial markets. One, historically the youngest and computer intensive by nature, consists of simulating the behaviour of many agents interacting through a financial market, buying and selling the same assets repeatedly in time. This approach is sometimes referred to as the "artificial stock market" model or the heterogenous agents model. By using such methods, [1] showed that such a market creates price paths similar to those issuing from $G A R C H$-type processes: non stationary, heavy tailed processes. More recently, [24] presented a model where all the agents have access to the past values of the price of a given asset, and trade according to one of two trading strategies: a naive cost free strategy, or a costly rational strategy. At any point in time 
agents may decide to use one or the other strategy. Through simulation, and also through analytic demonstration relying on chaos theory, they show that the added degree of freedom of allowing agents to select their model generates highly complex, completely unpredictable stock prices dynamics. Even so, within the specific model they constructed, they show that there exists a recurring cycle in term of pricing model usage: the system oscillates between a state where all the agents use the naive strategy and a state where all the agents use the rational strategy. This type of oscillations is found in different types of artificial stock markets, but also in social experiments [22, 23, 37, 38]. In this light, it is interesting to consider artificial stock markets as repeated games where agents compete to choose the best strategy rather than a method to build lifelike price processes.

From this point of view, heterogenous agent models resemble cascade models [3-5]. Indeed, in this type of models, agents are also sequentially faced with the same choice between different strategies. All cascade models share the same setup where agents have private information on the quality of the strategies, but also knowledge of the choice made by the people before them in the sequence. $[2,3,13]$ showed that in such a situation, after enough time, all the agents will choose the same strategy, hence the name of cascade. Moreover, this equilibrium is stable.

Hence, even if heterogenous agents models and cascade models share the same approach, there is one major difference between them: in the heterogenous agents models, the state of all the agents using the same strategy is unstable, allowing for oscillations, while in the cascade models it is stable. This difference in results can be explained by the central difference between the two models: in the heterogenous agents model, agents do not have perfect knowledge of the strategies employed by the other agents, but only an approximation of it through an educated guess made from the past realizations of the price, while they do have this knowledge in the cascade model. This lack of precise information causes the instability of the state where all agents use the same model in the heterogenous agents model.

Interestingly, both heterogenous agents and cascade models, beside sharing the same approach, also share the same aim of explaining the inefficiencies of financial markets. They both show that even if each agents are rational, the resulting financial market might not be efficient, or more precisely that the price of a financial asset might not be representative of the sum of the information present in the market, as classical efficiency theory describes it. This means that the quality of the information is the central parameter of both the heterogenous agents and the cascade models: this at both the agent level and the market level. There are two types of information to consider: the information each agent possesses on other agents' behavior and the information the current price contains. So the key concept to be studied is the propagation of the information: from the agent level to the market level, from the market level to the agent level and in-between agents.

This was already noted by R. Shiller; in [35] he went even further stating that to explain the propagation of the information one should study the "conver- 
sation" of the group. The concept of "conversation" of a group is rich, but can be summarised as follows: it is the means agents use to transform and interpret the information they receive as well as communicate it to other agents, much as language is our means of expressing and interpreting ideas and concepts. Clearly, both the cascade and the heterogenous agents models present specific types of "conversation" between agents, and this difference in the quality of the "conversation" leads to different equilibriums. In this paper we intend to offer a framework allowing for any kind of "conversation" between agents so as to discuss at the most general level the quality of a system where agents in a market interact through "conversation", but also to provide a powerful tool for research and to avoid any parametric specification pitfalls.

In our approach, to mimic the concept of "conversation", we assume that agents receive information regarding a firm and then process this information in order to acquire a point of view regarding the corresponding share price. We refer to the first type of information as input information and the other, the agents' points of view, as output information. This has two consequences.

The first is that, under our assumption, even if we make the hypothesis that markets aggregate information efficiently, the information according to which the price is set is the sum of all the agents output information, which is different from the aggregate of all the input information. This is what we referred to as the limited efficiency of financial markets. The equivalence of the input and output information could be claimed only if agents processed information perfectly. This means that agents would not only have to be perfectly rational but also infinitely knowledgeable about how the economy works. This is an assumption we do not make.

The second consequence is that each agent possesses a black box that transforms input information into output information: we call this device "model". This black box models two aspects of the agent. The first is how the agent perceives the information, a topic of concern to behavioural finance and psychology. The second is how the agent processes the information: for instance a set of formulas or a mathematical model. It is however best understood as a mathematical function, that takes as a parameter all the input information, and that returns the output information: a predicted distribution of the price.

We discuss the nature of the subsequent limited efficiency using two concepts: information efficiency, i.e., how well the price represents the sum of all the input information, and language efficiency, i.e., how well agents interpret the current price and infer the sum of all the input information. The former is close in meaning to the classical definition of market efficiency, while the latter is an agent-oriented efficiency: how well does the resulting price provide useful information to all the agents. Mirroring these two concepts, we also use the terms information risk and language risk to denote, respectively, cases where information efficiency and language efficiency are lacking. These two types of efficiency and risk are usually not differentiated; as we will see, the duality between the two becomes apparent in our framework only because we do not make assumptions regarding the models used. We conduct our analysis by studying two cases of our framework: the case where all the agents use the same model 
and the case where multiple models exist.

First we present the mathematical framework used to conduct our analysis. We then present the properties of the constructed framework and conclude with a simulation study.

\section{The Framework}

Most of these definitions concern a specific firm. For simplicity, we call this firm A.

\section{$2.1 \quad$ Definitions}

- Let $S$ be the set of possible values of an $A$ related asset.

- Let $\sigma_{S}$ be a $\sigma$-algebra over $S$.

- Let $\mathcal{F}\left(\sigma_{S}\right)$ be the set of measures on $\left(\sigma_{S}, S\right)$.

- Let $\left\{F_{u}\right\}_{u \leq t}$ be an increasing family of sets containing for each $u$ all the possible values concerning $A$ at that time.

- Let the $\sigma_{F_{t}}$ be $\sigma$-algebras over $F_{t}$ such that $\left\{\sigma_{F_{u}}\right\}_{u \leq t}$ be a filtration.

- Let $\mathcal{F}\left(\sigma_{F_{t}}\right)$ be the set of measures on $\left(\sigma_{F_{t}}, F_{t}\right)$.

- Let $\left\{W_{u}\right\}_{u \leq t}$ be an increasing family of sets containing for each $u$ all the possible values of the parameters concerning the economy at that time.

- Let $S$ and $F_{t}$ be such that: $S \subset W_{t}, F_{t} \subset W_{t}, S \cap F_{t}=\varnothing$.

- Let $\mathcal{M}$ be a set of functions $m$ such that $m: \mathcal{F}\left(\sigma_{F}\right) \rightarrow \mathcal{F}\left(\sigma_{S}\right)$.

- Let $H$ be a set. Define $\Phi_{t}: H \rightarrow\left(\mathcal{F}\left(\sigma_{F_{t}}\right), \mathcal{M}\right)$.

The following further explains our premise with examples of these mathematical definitions.

\section{$2.2 \quad$ Examples}

- $W_{t}$ and $F_{t}$ could be equal to $\mathbb{R}^{d_{t}}, d_{t}$ being the number of relevant economic variables at time $t$, concerning the whole economy for $W_{t}$, and concerning $A$ for $F_{t}$. Similarly $S$ is all the possible values of $A$ 's stock. Usually $S=\mathbb{R}^{+}$. $F_{t}$ is increasing with $t$ because it contains the past realisations of the price among other possible new variables of interest.

- The elements of $\mathcal{F}\left(\sigma_{F_{t}}\right)$ are information regarding the firm $A$ at time $t$. They are probability distributions over the value of $A$ 's relevant economic variables at that time. Likewise the elements of $\mathcal{F}\left(\sigma_{S}\right)$ are distributions over $S$ and as such are information on the price of the asset. 
- $\mathcal{M}$ is the set of "models" $m$, and - as defined in the premise $-m$ maps information on $A$ to information on the asset's value ${ }^{1}$.

- $H$ is the set of agents. At time $t$, each agent $h \in H$ possesses information and model $\Phi_{t}(h)=\left(i_{h}^{t}, m_{h}^{t}\right)$.

We will now put the framework into action. As our analysis is mostly static we will omit the index $t$ until part 4.2.

\subsection{Price Construction}

We now assume that the market efficiently aggregates information, i.e., that the price reflects all of the information present in the market. Let us first define the operator $\oplus$ as an application that maps a collection of distributions to one distribution representative of all of them. We make no assumptions regarding $\oplus$ besides its efficient information aggregation property, as this loose definition is sufficient for our purpose. Next, we define the sum of all information present in the market as $\oplus_{h \in H} m_{h}\left(i_{h}\right)$. The market then generates the following price ${ }^{2}$ :

$$
\mathbb{E}\left[P \mid \bigoplus_{h \in H} m_{h}\left(i_{h}\right)\right] .
$$

This allows for the price to reflect all of the output information present in the market, and in so doing to respect the efficient information aggregation hypothesis.

The mathematical framework we just presented has the advantage of being sufficiently flexible to contain a vast majority of the models used in the financial and behavioural literatures, and also allows for agents to transform the information they receive before interacting with the financial market. For instance, consider the following parallel with conventional financial models: in these models, $i_{h}$ is a Dirac mass, a specific value that agent $h$ believes is the true value of the information, $m_{h}\left(i_{h}\right)$ is then a prediction of the value of the price that the agents computes with $i_{h}, \oplus_{h \in H} m_{h}\left(i_{h}\right)$ is the population distribution of all such predictions and the price is the mean of this distribution. In the same fashion, our framework is coherent with all of the works we cite.

\section{$3 \quad$ Limited Efficiency}

By making the simplifying assumptions that the information is homogeneously distributed over agents using different models and that all models are used, we

\footnotetext{
${ }^{1}$ We choose to represent models as deterministic functions of the input information, defining them as random variables of parameter the input information is also possible, for instance to model for agents' erratic behaviour or errors. Such a modification would not alter our results.

${ }^{2} \mathrm{~A}$ more general construction of the price is possible; for instance, it could be a random variables of distribution $\oplus_{h \in H} m_{h}\left(i_{h}\right)$ or defined through any distribution operator $\Theta$ : $\Theta\left(\oplus_{h \in H} m_{h}\left(i_{h}\right)\right)$, for example the median.
} 
can rewrite formula (1):

$$
\mathbb{E}\left[P \mid \bigoplus_{m \in \mathcal{M}} m(\mathcal{I})\right]
$$

Where $\mathcal{I}=\bigoplus_{h \in H} i_{h}{ }^{3}$. Using this formula we will now draw conclusions concerning efficiency.

Comparing formula (2) to the results of classical market efficiency (more precisely to $\mathbb{E}[P \mid \mathcal{I}]$ ), one can see that in our framework the market can only be considered informationally limitedly efficient because it is not efficient relative to the actual information possessed by agents, $\mathcal{I}$ or input information. Instead it is efficient relative to $\bigoplus_{m \in \mathcal{M}} m(\mathcal{I})$, a transformation of $\mathcal{I}$. That markets can be structurally limitedly efficient as a consequence of a agent's information processing is discussed in $[35,36]$. These works argue that our communication patterns and culture form a filter through which the input information is first processed before being fed to the markets, fact that affects the quality of the output information. Our framework permits to formalise this result: the filter through which the input information is precessed is $\bigoplus_{m \in \mathcal{M}} m$.

A situation where a perfect model is used by all agents would allow for classical market efficiency. This situation would turn formula (2) into one resulting in classical market efficiency. However, the concept of a perfect model in our framework is paradoxical. To say that a model is better than another requires some set of criterion that defines the desired relationship between the input information and the price, but such criterion is a "model", hence the paradox. Indeed, in our framework the link between the information and the price exists solely through the "models" and does not aim to respect predefined standards. As such, all models are neither perfect nor imperfect: they represent a relationship between the input information and the price.

Because our framework is structurally limitedly efficient, a characteristic that cannot be circumvented, we will focus on the quality of the subsequent limited efficiency associated with specific kinds of $\oplus_{m \in \mathcal{M}} m$.

\section{Balance of Efficiencies}

To understand the nature of limited efficiency and the consequences it has on the behaviour of the market, we will study the following two cases: the one model and the multiple model case. Each offers one specific kind of efficiency that the other does not. The single model case allows for language efficiency but not information efficiency while the multiple model case allows for information efficiency but not language efficiency.

\footnotetext{
${ }^{3}$ This is an abusive notation, as it is possible that $m\left(\oplus_{h \in H} i_{h}\right) \neq \oplus_{h \in H} m\left(i_{h}\right)$. However, this does not affect our results.
} 


\subsection{The One Model Case}

If there exist only one model, formula (2) can be rewritten as:

$$
\mathbb{E}[P \mid m(\mathcal{I})]
$$

This expression can be further simplified by defining $m(\mathcal{I})$ as a distribution over $S$ so the asset's price is equal to:

$$
\mathbb{E}[m(\mathcal{I})]
$$

\subsubsection{Self Fulfilling Powers}

Formula (3) shows that if the considered model was to change so would the resulting price. This implies that the used model has self fulfilling powers. For example, suppose that event " $e$ " occurs and is witnessed by all agents. If the model predicts that " $e$ " causes the price to rise, this prediction will be verified. In general, one could expect the main properties of the model to be verified. The most troubling case would be " $m=C^{\text {te" }}$ disconnecting the price and the economy, most likely leading to a severe economic crisis [20, 30]. We distinguish three risks associated with a reliance on only one model. A simple example illustrates each.

Information Decay The first risk is relative to the fact that the model $\mathrm{m}$ may omit relevant variables. " $m=C^{t e}$ ", for example, is the most extreme case of information decay. Consider the following:

- $S=\mathbb{R}^{+}$. Agents possess information concerning $l$ different parameters related to $A$ : $i=\left(i_{1}, \ldots, i_{l}\right)$. They also posses information on the real interest rate $r$ on the company $A$ 's income $I$ and on its debt $D$ so that $F=\mathbb{R}^{l+3}$.

- The model $m$ is $m=m(i, r, I)$, but it is not a function of the debt $D$.

Given a $r$ and a $I$, if $D$ is large it is possible that $I<r \cdot D$ and $0<\mathbb{E}[m(i, r, I)]$. In this case $A$ is bankrupt but the asset's price is still positive, and investors will lose all their capital as $A$ fails to pay its debt. Other more subtle cases of information decay can be found: mental compartment, overconfidence, and gambling behaviour ... [35, 36].

Information Misuses The second risk is contrary to the first and is the case where the model $m$ regards a variable as relevant when it should not. Consider the following:

- $S=\mathbb{R}^{+} . A$ is a corn related company. Agents possess information concerning $l$ different parameters related to $A: i=\left(i_{1}, \ldots, i_{l}\right)$. They also posses information on the expected number of sunspots $n$ so that $F=\mathbb{R}^{l+1}$. 
- The model $m$ is such that $\mathbb{E}[m(i, n)]$ is increasing in $n$. The basis for this model is that an increase in the number of sunspots $n$ will increase the quality of the corn. However, this is untrue.

In this example, the share price depends on $n$ although the actual quality of the corn does not. Hence, the price possesses a random component when it should not. If the number of expected sunspots dramatically increases, mechanically the price of the corn will increase even if there is no excessive demand for it, creating a bubble. More subtle cases of information misuse can be found in either Sunspot literature or in studies on magical thinking [9, 14, 26, 41].

Systemic Cascades Thirdly, structural problems may occur when all agents rely on a single model based on other agents behaviour. Indeed, such situation can lead to self sustaining loops. Consider the following example:

- $S=\{0,1\}$. Agents possess information concerning $l$ different parameters related to $A: i=\left(i_{1}, \ldots, i_{l}\right)$ and exact information on the number of agents who bought one share of $A$ in the past $k$. Thus, $F=\mathbb{R}^{l+1}$

- The model $m$ is $m(i, k)=\mathbf{1}_{\left\{m_{1}(i)+m_{2}(k) \geq \frac{1}{2}\right\}} \cdot m_{1}$ and $m_{2}$ are two subfunctions such that $m_{2}$ is increasing in $k$, and $m_{1}$ is positive.

Let $k^{*}$ be such that $m_{2}\left(k^{*}\right)=\frac{1}{2}$. In this example, if $k^{*}$ people bought a share of $A$ the price remains forever at 1 despite the other parameters. Similar results can be found in the Cascade-Herd literature [2-5, 13].

These arguments show that the one model case is economically unstable. Even though the information is correctly aggregated, the information according to which the price is set is unreliable. As described above, using only one model $m$ presents real risks, associated with the quality of its transformation of the input information. Consequently, we say that the market's information efficiency is low, or conversely that its information risk is high.

\subsubsection{From Model To Language}

As previously shown, the capacity of agents to infer from the price information present in the market is a possible escape from the no-trade theorems [7, 31] and also insures liquidity $[6,10,33]^{4}$. In the one model case, each agent can use $m^{-1}$ to infer information from the market price as well as the price proposed by any other agent in a trade. Consequently, $m$ becomes a language ${ }^{5}$ in the sense that it transmits meaning, $i_{h}$, through a representation $m\left(i_{h}\right)$ that can be interpreted with $m^{-1}$. This ensures that the market efficiently communicates information to all agents and vice versa.

\footnotetext{
${ }^{4}$ Please note that this literature often shows that the readability of other agent's information also leads to bubbles caused by informational cascades. We consider this to be explained by the arguments exposed in 4.1 .1 and not by the readability itself.

${ }^{5} \mathrm{~m}$ is not strictly speaking a language because it does not possess a semantic structure or grammar [28].
} 
Using models as language is a everyday occurrence in financial markets: volatility implies the use of Black \& Scholes's formula, yield implies the use of discounted cash flows, and correlation implies the use of Gaussian copulas or Gaussian distribution. Furthermore, tools such as the "implied volatility" and the "smile" in the option market are effectively using the inverse of Black \& Scholes's formula $[8,11,15,19]$.

In the one model case, we consider that the use of $m$ as a language and the ensuing readability of the market indicates that there is proper information propagation between agents, and that it returns to the market price its value as an economical indicator. Consequently, we say that in the one model case the market is language efficient. However, each agents uses a special modification of the Black \& Scholes formula, interpolates the yield curve differently and uses a different mixtures of copulas. We will now consider the multiple model case.

\subsection{The Multiple Model Case}

This section studies the multiple model case. We consider this case not only for the reasons explained above, but also because each agent may possess a particular bias or irrational behaviour. Agents may act with different purposes inside a market: for example, an agent may buy a share because he expects its price to rise or it presents a good diversifying property for his portfolio. He might also be looking for ownership of the company. More generally, the purchase of an asset can be motivated by varied strategies each implying a different "model". Taking these issues into consideration, we will not specify the models used and try to obtain general results concerning language efficiency and information efficiency in this case.

Here the price is set by formula (1):

$$
\mathbb{E}\left[P_{t} \mid \bigoplus_{h \in H} m_{h}^{t}\left(i_{h}^{t}\right)\right] .
$$

\subsubsection{Information Efficiency}

As noted above, the multiple model case is limitedly efficient. Although, the multiplicity of models make the risk described in 4.1.1 less likely. For the system to show self fulfilling properties it would require all used models to share the same failing. The probability of such an event decreases with the number of models $[12,25]$. The same can be said about the three risks in 4.1.1: information decay, information misuses and systemic cascades. In this case the market is more informationally efficient than the one model case because it has lower information risk.

\subsubsection{Readability}

However, in the presence of multiple models using $m$ as a language becomes less efficient. In a trade agents do not know which model uses the counterpart, nor

is the global effect of $\oplus_{h \in H} m_{h}^{t}$ known. In this case, using $m_{h}^{t-1}$ only provides 
an approximation. The literature address this question through two different cases.

Fixed Model This is when agents are bound to one specific model associated with their cognition. Here, the diversity of approachs increases the "composition uncertainty" of the market making cascades more probable [2]. Also, assuming a parametric representation of the models in a framework similar to ours, the market evolution can be described by a system of ordinary differential equations [17, 18, 21, 25]. Consider the following example:

- $S=\mathbb{R}^{+}$. At time $t$ agents know $\bar{i}_{t}$ the past realisations of $l$ different information concerning $A, i_{t}=\left(i_{1 t}, \ldots, i_{l t}\right)$ and $\bar{p}_{t}$ the past realisations of the price $p_{t}$, so that $F_{t}=\mathbb{R}^{t \cdot(l+1)}$.

- We place ourselves in an updating expectation framework. Each agent $h$ holds a model $m_{h}$ as true, and he estimates this model with the estimator $\underline{m}_{h}$ on the available dataset at time $t-\bar{i}_{t}$ and $\bar{p}_{t}-$ yielding the estimate $m_{h, t}$. Then, at time $t$, agent $h$ predicts that the price at time $t+1$ will be $p_{h, t+1}^{e}$ as follows:

$$
\begin{aligned}
& p_{h, t+1}^{e}=m_{h, t}\left(\bar{i}_{t}, \bar{p}_{t}\right), \\
& m_{h, t}\left(\bar{i}_{t}, \bar{p}_{t}\right)=t^{-1} \sum_{s=1}^{t} \underline{m}_{h}\left(i_{s}, p_{s}\right)=\frac{t-1}{t} m_{h, t-1}\left(\bar{i}_{t-1}, \bar{p}_{t-1}\right)+t^{-1} \underline{m}_{h}\left(i_{t}, p_{t}\right) .
\end{aligned}
$$

Where $\underline{m}_{h}$ and $m_{h, t}$ are linear forms such that $\underline{m}_{h}: \mathbb{R}^{l+1} \rightarrow \mathbb{R}$ and $m_{h, t}$ : $\mathbb{R}^{t \cdot(l+1)} \rightarrow \mathbb{R}$. The resulting price is ${ }^{6}$ :

$$
p_{t+1}=\frac{1}{|H|} \sum_{h \in H} p_{h, t+1}^{e}=\mathbb{E}_{H}\left[p_{h, t+1}^{e}\right]=\mathbb{E}_{H}\left[m_{h, t}\left(\bar{i}_{t}, \bar{p}_{t}\right)\right] .
$$

- By merging the previous formulas we obtain:

$$
m_{h, t}\left(\bar{i}_{t}, \bar{p}_{t}\right)=\frac{t-1}{t} m_{h, t-1}\left(\bar{i}_{t-1}, \bar{p}_{t-1}\right)+t^{-1} \underline{m}_{h}\left(i_{t}, \mathbb{E}_{H}\left[m_{h^{\prime}, t-1}\left(\bar{i}_{t-1}, \bar{p}_{t-1}\right)\right]\right) .
$$

Assuming that the $i_{t}$ are identically distributed of law $I$ and mean $i$, asymptotically we have:

$$
\begin{aligned}
\forall h \in H \quad \Delta_{t} m_{h, t} & =\underline{m}_{h}\left(i, \mathbb{E}_{H}\left[m_{h^{\prime}, t}\left(\bar{i}, \mathbb{E}\left[\bar{p}_{t}\right]\right)\right]\right), \\
\Delta_{t} p_{t} & =\mathbb{E}_{H}\left[\underline{m}_{h}\left(i, \mathbb{E}_{H}\left[m_{h^{\prime}, t}\left(\bar{i}, \mathbb{E}\left[\bar{p}_{t}\right]\right)\right]\right)\right] .
\end{aligned}
$$

so that the system is driven by ordinary differential equations of order $|H|+$ 1. In such cases a small variation in parameter can have large and complex effects on the price [32, 34]. Then, as the number of models increases, so does the dimension of the phase space, making the equilibrium path less and less predictable.

\footnotetext{
${ }^{6}$ We use the shorthand: $\frac{1}{|H|} \sum_{h \in H}=\mathbb{E}_{H}$ while $\mathbb{E}$ denotes the distribution expectation. As said in 2.3, using any other distribution operator to define the price would not affect the results.
} 
Free Model This is the case when agents can freely change models. For example, they can use a different set of formulas to describe the economy. Assuming a parametric form for the used models in a framework similar to ours, the resulting time-series of the asset price is chaotic [22-24]. Consider the following:

- $S=\mathbb{R}^{+}$. At time $t$ agents know $\bar{p}_{t}^{d}$, the $d$ past realisations of the price $p_{t}$ : $\bar{p}_{t}^{d}=\left(p_{t}, p_{t-1}, \ldots, p_{t-d}\right)$ so that $F_{t}=\mathbb{R}^{d}$.

- There are $n$ models used: $m_{1}, \ldots, m_{n}$. For all $k \in \llbracket 1, n \rrbracket, m_{k}: \mathbb{R}^{d} \rightarrow \mathbb{R}$. To each model $k$ is associated a performance measure $U_{k}$, which is a function of $\bar{p}_{t}^{d}$. At time $t$, agent $h$ measures $U_{k}\left(\bar{p}_{t}^{d}\right)$ with noise, and we denote $U_{h_{k, t}}$ as this measure:

$$
\forall h \in H, \forall k \in \llbracket 1, n \rrbracket, U_{h_{k, t}}=U_{k}\left(\bar{p}_{t}^{d}\right)+\epsilon_{h_{k, t}} .
$$

Where the noises $\epsilon_{h_{k, t}}$ follow the Gumbel type I extreme value distribu$\operatorname{tion}^{7}$ and are independent between agents. According to classical multinomial choice theory [29]:

$$
\mathbb{P}(\text { Agent } h \text { using model } k \text { at time } t)=\frac{e^{U_{k}\left(\bar{p}_{t}^{d}\right)}}{\sum_{k^{\prime}=1}^{n} e^{U_{k^{\prime}}\left(\bar{p}_{t}^{d}\right)}} .
$$

We define as $\mu_{k, t}$, the proportion of agents using model $k$ at time $t$. Assuming that $|H|$ is large enough:

$$
\forall k \in \llbracket 1, n \rrbracket, \quad \mu_{k, t}=\frac{e^{U_{k}\left(\bar{p}_{t}^{d}\right)}}{\sum_{k^{\prime}=1}^{n} e^{U_{k^{\prime}}\left(\bar{p}_{t}^{d}\right)}} .
$$

- Since the resulting price is the mean of agents' expected prices, the market can be described by the following set of equations:

$$
\begin{aligned}
\forall k \in \llbracket 1, n \rrbracket, \quad \mu_{k, t+1} & =\frac{e^{U_{k}\left(\bar{p}_{t+1}^{d}\right)}}{\sum_{k^{\prime}=1}^{n} e^{U_{k^{\prime}}\left(\bar{p}_{t+1}^{d}\right)}} \\
p_{t+1} & =\sum_{k^{\prime}=1}^{n} \mu_{k^{\prime}, t} \cdot m_{k^{\prime}}\left(\bar{p}_{t}^{d}\right) .
\end{aligned}
$$

System that can be re-written:

$$
\left(\bar{p}_{t+1}^{d}, \mu_{1, t+1}, \ldots, \mu_{n, t+1}\right)=\varphi\left(\bar{p}_{t}^{d}, \mu_{1, t}, \ldots, \mu_{n, t}\right) .
$$

This demonstrates that in this case the market is driven by a $(h+n)$-dimensional difference equation. This type of system leads to mathematical chaos, and the resulting time series are highly unpredictable. In practice, the price trajectory becomes less and less predictable as the number of models increases, and laboratory experiments confirm this [1, 37, 38].

\footnotetext{
${ }^{7}$ The Gumbel type I extreme value distribution function is: $F(x)=e^{-e^{-x}}$.
} 
In both cases the market price becomes unpredictable and less informative to the agents as the number of models increases because its interpretation becomes ambiguous [16]. This causes multiple market issues: cascades, bubbles, crashes and fat-tails. In the multiple model case, the market has a large language risk because it is not readable by the agents and the price loses its use an economic indicator.

\subsection{Discussion}

Our results can be summarised as follows:

$$
\begin{array}{llll}
\text { Single Model } & \Rightarrow \text { language efficiency } & \mathcal{G} \text { information risk, } \\
\text { Multiple Models } & \Rightarrow \text { language risk } & \mathbb{G} \text { information efficiency. }
\end{array}
$$

It becomes apparent that there is a compromise between language efficiency and information efficiency in our framework, and that this compromise is controlled by the number of different models used. Paradoxically, even though more information is present in the multiple model case it is not accessible to the agents. Whereas, in the one model case, information can be inferred by agents but this information is unreliable. Overall there is an incompressible part of inefficiency, and the only control agents posses is over the quality of this inefficiency by controlling the number of models present. We now turn to the second part of this paper where we will estimate how a group of agents would naturally set these efficiency compromises.

\section{A Small World}

Here we will present a simulation of the above described framework in the multiple free model case. We are interested in how a group of agents naturally set the balance between language efficiency and information efficiency. We assume that agents are unaware of the issue we are discussing and are looking for a natural equilibrium.

\subsection{The Model}

- There are $N$ agents. (According to our previous notation: $N=|H|$.)

- Each agent possesses one of $l$ possible bits of information: $F=\left\{i_{1}, \ldots, i_{l}\right\}$. Agents possess $i_{k}$ with probability $p_{k}$. Each bit of information predicts that an event will occur with probability one. At the end of their turn, agents can verify the validity of these bits of information.

- There are $n$ "models": $m_{1}, \ldots, m_{n} . \forall s, k m_{s}\left(i_{k}\right)$ is a Dirac mass so that: $m_{s}\left(i_{k}\right) \in \mathbb{R}$. Each agent uses only one model and is unaware of which model the other agents are using. 
- The agents are risk neutral. They will trade with whomever proposes a different price than theirs, selling and buying according to their prices relative position. They trade only once.

- The market is cleared by each agents finding a counterpart. The resulting price is the one that maximise the number of exchanges.

- After the price is set, agents will change models if they had correct information and still predicted the wrong price. They draw uniformly from the set of models.

The system is entirely defined by a $n \times l$ matrix $M$ containing the number of agents in all possible cases: $\left(m_{s}, i_{k}\right) \forall s, t \leq n, l$. As we do not which to specify the information nor the models used here, we draw one permutation uniformly over $\mathfrak{S}(n \cdot l)$ to decide the order of the prices. In our framework this sufficiently sets the equilibrium price.

After the price has been selected, agents can verify whether they had true or false information. Agents with wrong information do not change "models" because they have no reason to. Agents with good information but a wrong price change models, since they have proof that their model predicted the wrong price with good information. We then iterate the process.

\subsection{Analysis}

We use permutations to set the equilibrium because it permits to avoid giving any specific parametric form to the used models. Indeed, we are interested in the behaviour of the number of models in use outside any specifications. Moreover in [22-24] the specific parametric forms given to the models tend to be pro-cyclical or complementary, scenarios that may affect our results.

The aim in using Dirac masses is simplicity. It permits to make agent change models after failing once instead of requiring them to fail enough times to establishing a statistically significant rate of failing. We allow this because our analysis is not concerned with the intermediary steps.

This model and subsequent behaviour is similar to that of complex systems and evolutionary games $[27,39,40]$. It is also a Markov process. However, due to the use of random permutations, the transition matrix dimension of the process is in the order $(n \cdot l) ! \sum_{k=1}^{n \cdot l}\left(\begin{array}{c}N \\ k\end{array}\right) B_{k}$ so that its computation involves $\mathcal{O}\left(n !(n \cdot l) ! N ! \sum_{k=1}^{n \cdot l}\left(\begin{array}{c}N \\ k\end{array}\right) B_{k}\right)$ operations ( $B_{k}$ are Bell's numbers). Although closed formula can be produced, to compute the distribution of $M$ at the $r^{t h}$ step requires $\mathcal{O}\left(n !^{r} \cdot N !^{n \cdot l}\right)$ operations. This motivates the use of simulations that require only $\mathcal{O}(r \cdot n \cdot l \cdot N)$ operations, thereby making it more computationally efficient. All computations are done in R.

\subsection{Results and Discussion}

According to this simulation study there is a cycle between two states. The first state is the "chaos" state where there is no selected "model", and the second is 


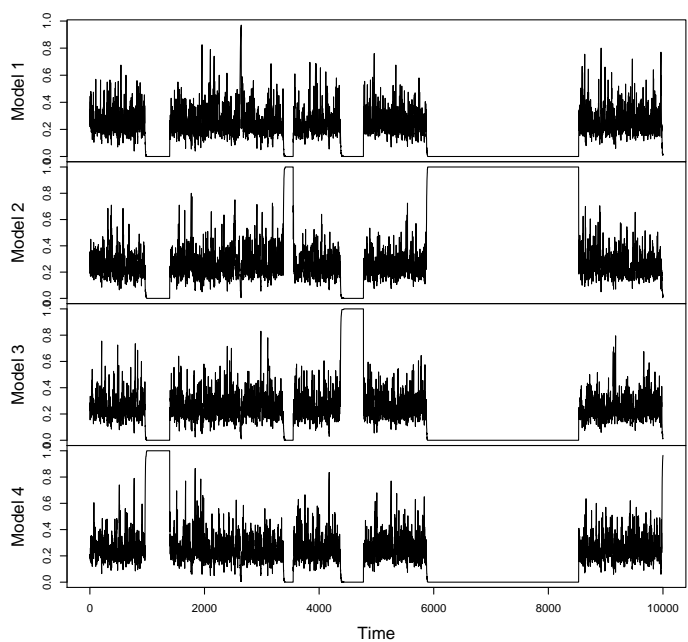

Figure 1: Proportion of agents using each model in an example of 1000 steps dynamic with 4 models, 3 bits of information, 500 agents and a probability of having true information of 0.6 .

the "cascade" state where there is. They correspond respectively to the moving and flat lines in Figure 1. As is done in [39], we simplify the system to a twostate model, which is similar to a two state space Markov proces. This system is entirely determined by the following two probabilities: $p_{C h a o s}$, the probability of remaining in the chaos state, and $p_{\text {Cascade }}$ the probability of remaining in the cascade state. We plot estimations of $p_{\text {Cascade }}$ and $p_{\text {Chaos }}$ in Figure 2.

According to 4.3, these results imply that the market oscillates between an information efficient state, the "chaos" state where multiple models are present, and a language efficient state, the "cascade" state where only one model is present. In our simulation the higher the probability of having correct information, the smaller the cycle's frequency. Increasing the number of models $(n)$, the number of agents $(N)$ or the number of bits of information $(l)$ does not alter this. Still, according to 4.3, this would indicate that the gain of information efficiency linked to an increase in the probability of having true information is automatically compensated by an increased information risk through a more frequent and stable cascades toward a single model. Overall the market tends to be stable informational efficiency-wise.

This answers our question on how the number of models is naturally regulated by the agents. It is fixed to keep the global informational efficiency constant. The fact that, unknown to the agents, the market stabilises itself to a risk neutral state coherent with the agents' preferences is reminiscent of the "invisible hand" or market efficiency, but at another level. 
$p_{\text {Cascade }}$

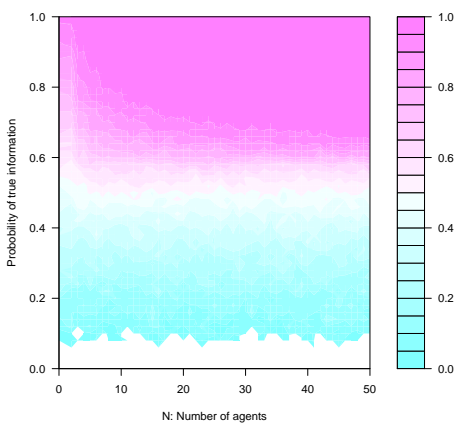

$1-p_{\text {Chaos }}$

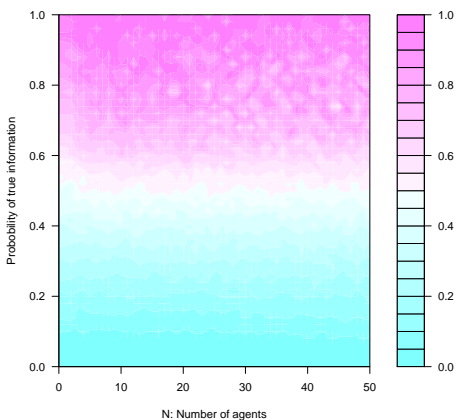

Figure 2: $p_{\text {Cascade }}$ and $1-p_{\text {Chaos }}$ in a two-model system, each point estimated with 500 simulations of 1000 steps.

\section{Conclusion}

We conclude that if one assumes that agents transform the input information before interacting with a financial market, the ensuing market will be limitedly efficient. This independently of any assumption on how agents transform the input information. Using the new concept of "model" and a simple yet very flexible mathematical structure, we put into perspective the dual quality of limited efficiency: that of a balance between information efficiency and language efficiency. Furthermore, we have shown that agents control this balance through the number of different models in use. Fewer models increase the language efficiency while more models enhance the information efficiency. In a simulation study we have shown that the quality of the information influenced the number of models present. If given more reliable information, a group of risk neutral agents tend to reduce the number of models used to increase the resulting language efficiency of the market.

Within real markets there are none that fall strictly within the single model case. However, in practice, long term financial models broadly share the same characteristics. According to our findings, long term markets should be broadly stable and readable but prone to extreme variation, which is the case. Conversely, there is much more heterogeneity within short term models so that the short term market should appear random. This is a widely accepted fact. Applying our result to the still recent financial crisis provides the following interpretation: agents assumed the information present to be reliable, which led to the widespread use of a single model that weakened the economy in the face of wrong information (subprimes) and contributed to systemic issues (the credit crunch).

These results also give interesting insight into what role economics and finance play in the market. In our framework, the models they produce define the behaviour of the market, however, in practice these models intend to accurately 
represent the market. This paper presents a new perspective where one perfect model or underlying mechanism to be modelled does not exist but instead presents the market as driven by agents' "models". However our framework allows for the existence of a set of models that would make the economy behave as we want. Hence, economic and financial disciplines could aim to produce "models" that would answer the dual criteria of defining the objective behaviour of the market while inducing a price-setting process coherent with this objective behaviour. Lacking this coherence, the model would generate instability most likely leading to a crisis and its abandonment.

However, this raises the question of how we want the markets to behave. Should gains be possible only if one has informational advantage? Or should it be a place where speculation is possible? We should note that in our framework people in favour of speculation have the advantage. If they wish for the market to remain random they only need to act according to a different model to reduce the overall language efficiency.

Further perspectives would be concerned by the following aspects of the framework: the information aggregation $(\oplus)$, which we did not describe, and how the adoption of models propagate within a group. Both would need to represent the agent-to-agent relationship and would most likely rely on a network linking them. Finally, as the mathematical framework is simple and flexible it allows for possible extensions to other fields, especially group decision making.

\section{Acknowledgments}

During the preparation of this paper, we benefited from helpful discussions with Miriam Maugis, Paul Daladier, Arun Chandrasekhar, Fabien Gensbittel, Vincent Pons. Of course, any remaining errors are entirely ours.

\section{References}

[1] Arthur, B., Holland, J., LeBaron, B., Palmer, R., Tayler, P., 1996. Asset Pricing Under Endogenous Expectations in an Artificial Stock Market . Santa Fe Institute Publication.

[2] Avery, C., Zemsky, P., 1998. Multidimensional uncertainty and herd behavior in financial markets. The American Economic Review 88 (4), 714-748.

[3] Banerjee, A. V., 1992. A simple model of herd behavior. The Quarterly Journal of Economics 107 (3), 797-817.

[4] Bikhchandani, S., 1992. A theory of fads, fashion, custom, and cultural change as informational cascades. The Journal of Political Economy 100 (5), $992-1026$. 
[5] Bikhchandani, S., Hirshleifer, D., Welch, I., 1998. Learning from the behavior of others: Conformity, fads, and informational cascades. The Journal of Economic Perspectives 12 (3), 151-170.

[6] Brockman, P., Chung, D. Y., 2000. An empirical investigation of trading on asymmetric information and heterogeneous prior beliefs. Journal of Empirical Finance 7 (5), 417-454.

[7] Brunnermeier, M. K., 2003. Asset Pricing under Asymmetric Information. Oxford Scholarship.

[8] Canina, L., Figlewski, S., 1993. The Informational Content of Implied Volatility. The Review of Financial Studies 6 (3), 659-691.

[9] Cass, D., Shell, K., 1983. Do Sunspots Matter? Journal of Political Economy $91,193-227$.

[10] Chen, Q., Goldstein, I., Jiang, W., 2003. Price Informativeness and Investment Sensitivity to Stock Price. SSRN eLibrary.

[11] Christensen, B. J., Prabhala, N. R., 1998. The relation between implied and realized volatility. Journal of Financial Economics 50 (2), 125-150.

[12] Cremer, J., Garicano, L., Prat, A., 2007. Language and the Theory of the Firm. Quarterly Journal of Economics 122 (1), 373-407.

[13] Devenow, A., Welch, I., 1996. Rational herding in financial economics. The European Economic Review 40, 603-615.

[14] Duffy, J., Fisher, E. O., 2005. Sunspots in the laboratory. American Economic Review 95 (3), 510-529.

[15] Dumas, B., Fleming, J., Whaley, R. E., 1998. Implied Volatility Functions: Empirical Tests. Journal of Finance 53 (6), pp. 2059-2106.

[16] Easley, D., Maureen, O., 2010. Microstrutcture and ambiguity. The Journal of Finance.

[17] Evans, G., 1985. Expectational Stability and the Multiple Equilibria Problem in Linear Rational Expectations Models. Quarterly Journal of Economics 100, 1217-1233.

[18] Evans, G., Honkapohja, S., 2001. Learning and Expectations in Macroeconomics. Princeton University Press.

[19] Fabozzi, F. J. (Ed.), 2008. Handbook of Finance: Financial Markets and Instruments. J. Wiley Press.

[20] Farmer, R., 1999. Macroeconomics of Self-fulfilling Prophecy. MIT Press.

[21] Guesnerie, R., 2002. Anchoring Economic Predictions in Common Knowledge. Econometrica 70 (2), 439-480. 
[22] Hommes, C., 1996. Financial markets as nonlinear adaptive evolutionary systems. Quantitative Finance 1, 149-167.

[23] Hommes, C., 2002. Modeling the stylized facts in finance through simple nonlinear adaptive systems. Proceedings of the National Academy of Sciences 99, 7221-7228.

[24] Huang, H., Wang, D., Hommes, C., 2005. A robust rational route to randomness in a simple asset pricing model. Journal of Economic Dynamics and Control 29, 1043-1076.

[25] Huberman, B. A., Glance, N. S., 1993. Diversity and Collective Action.

[26] Jevon, W. S., 1878. Commercial crises and sun-spots. Nature 19, 33-37.

[27] Johnson, N. F., Smith, D. M., Hui, P. M., 2006. Multi-Agent Complex Systems and Many-Body Physics. Europhysics Letters 74, 923-929.

[28] Kello, C. T., 2004. Characterizing the evolutionary dynamics of language. TRENDS in Cognitive Science 8 (9), 392-394.

[29] Kenneth, T., 2003. Discrete choice methods with simulation. Cambridge University Press.

[30] Merton, R., 1968. Social Theory and Social Structure. New York: Free Press.

[31] Milgrom, P., Stokey, N., 1982. Information, Trade and Common Knowledge. Journal of Economic Theory 26, 17-27.

[32] Morris, S., Song-Shin, H., 2002. Social Value of Public Information. The American Economic Review 92, 1521-1534.

[33] Romer, D., 2006. Rational Asset Price Movements Without News. NBER Working Paper No. 4121.

[34] Sethi, R., Franke, R., 1995. Behavioural Heterogeneity Under Evolutionary Pressure: Macroeconomic Implications of Costly Optimisation . The Economic Journal 105, 583-600.

[35] Shiller, R. J., 1995. Conversation, information, and herd behavior. The American Economic Review 85 (2), 181-185.

[36] Shiller, R. J., 1999. Human behavior and the efficiency of the financial system. Handbook of Macroeconomics 1, 1305-1340.

[37] Sonnemans, J., Tuinstra, J., van de Velden, H., Homes, C., 2005. Coordination of expectations in asset pricing experiments. Review of Financial Studies 18, 955-980. 
[38] Sonnemans, J., Tuinstra, J., van de Velden, H., Homes, C., 2008. Expectations and bubbles in asset pricing experiments. Journal of Economic Behavior and Organization in press.

[39] Tarnita, C. E., Antal, T., Ohtsuki, H., Nowak, M. A., 2009. Evolutionary dynamics in set structured populations. Proceedings of the National Academy of Sciences 106 (21), 8601-8604.

[40] Traulsena, A., Hauert, C., De Silva, H., Nowak, M., Sigmund, K., 2009. Exploration dynamics in evolutionary games. Proceedings of the National Academy of Sciences 106 (3), 709-712.

[41] Woodford, M., 1990. Learning to Believe in Sunspots. Econometrica 58 (2), 277-307. 